\title{
Criteria in Radioisotope Detection of Venous Thrombosis
}

\author{
K. JEYASINGH, \\ H. I. GLASS, \\ N. H. HILLS, J. S. CALNAN
}

British Medical fournal, 1972, 3, 500-503

\section{Summary}

Forty-one patients out of a larger series undergoing elective surgery were examined for the presence of venous thrombosis in the legs by means of the ${ }^{225}$ I-fibrinogen technique. The radioisotope data were analysed by three different methods. The percentage uptake was calculated and, by the generally accepted difference of $15 \%, 22$ patients had evidence of thrombosis. The statistical index, which takes account of the errors due to radioactivity counting inherent in the percentage uptake calculation, indicated thrombosis in 36 patients. The relative uptake inder, which allows for the errors due to unequal distribution of radioactivity throughout the normal limb as well as the errors due to radioactivity counting, provided evidence of thrombosis in a total of 34 patients, compared with only 22 patients when assessed by the " $15 \%$ " criterion.

\section{Introduction}

The early detection of venous thrombosis in the legs with the use of fibrinogen labelled with radioactive iodine represents an appreciable advance in diagnostic technique. The method has now been in use for several years and is likely to become more widely used in the future. It is important therefore that it be applied in the most appropriate manner.

The test as first introduced (Atkins and Hawkins, 1956, 1968) has been modified in order to make it more easily performed (Flanc et al., 1968; Negus et al., 1968). All methods depend on the detection of an increased count rate due to the additional radioactivity at a site of fibrin deposition. The decision to be made is whether an observed increase in count rate is significant enough to justify the diagnosis of thrombosis, and the most commonly used criterion is that suggested by Negus et al. (1968). The count rate measured at different points along each leg is expressed as a percentage of the precordial count rate and is referred to as the "percentage uptake" for each point. A difference in percentage uptake of $15 \%$ or more between adjacent points on the same leg, or between corresponding points on the two legs, correlates well with the detection of thrombosis by phlebography; this percentage has therefore been accepted as a positive indication of a thrombus. This " $15 \%$ criterion" has been widely used and has the added attraction that a direct daily comparison between points is easily made.

Two serious criticisms may be levelled at this procedure. Firstly, it takes no account of the random errors involved in the estimation of a single count rate. Owing to decay of the radioisotope the count rate falls progressively and so the error rises on successive days; hence a $15 \%$ difference on the Royal Postgraduate Medical School and Hammersmith Hospital,
London W.12

K. JEYASINGH, B.sc., Physicist, Radioisotope Unit, Department of Medical Physics

H. I. GLASS, PH.D., F.INST.P., Head of Radioisotope Unit, Department of Medical Physics

N. H. HILLS, F.R.C.S., Research Fellow (Present address: St. Thomas's Hospital, London S.E.1)

J. S. CALNAN, M.R.C.P., F.R.C.S., Professor of Plastic and Reconstructive tenth day cannot have the same significance as a $15 \%$ difference on the first day. Secondly, the method takes no account of the variable anatomy of the legs from the point of view either of assymmetry of the two legs or of the unequal distribution of blood throughout a single leg. It has already been reported (Palko et al., 1964) that there is frequently a difference in count rate between corresponding positions on opposite limbs in the absence of thrombus formation. It is clear also that by using a fixed percentage criterion discrepancies may arise owing to the normal variation in count rate along the length of the limb.

In addition to these sources of error the accuracy of standardization of the technique by venography is open to serious doubt. Radiographic examination of the leg veins may well indicate the presence of relatively large thrombi but of ten fails to detect thrombi in small vessels or in veins which are not well filled with contrast medium. Interpretation of the radiographs in patients with small thrombi may be extremely difficult. Thus the use of a fixed percentage criterion based on phlebographic confirmation may be expected to underestimate the frequency with which thrombosis occurs.

The purpose of this paper is to present two further methods of analysis of count rates in which these errors are taken into account.

\section{Material and Methods}

Analysis was performed on data from 41 patients who were admitted for elective surgery under general anaesthesia. Selection was made so that about half the patients gave positive results on the basis of the $15 \%$ criterion.

All patients received $180 \mathrm{mg}$ potassium iodide by mouth 24 hours before operation and a further dose of $60 \mathrm{mg}$ twice daily for three weeks afterwards. Sodium iodide was given intravenously in a dose of $85 \mathrm{mg}$ once daily if the patient was unable to tolerate oral administration.

An injection of about $100 \mu \mathrm{Ci}$ of ${ }^{125}$ I-fibrinogen containing about $1 \mathrm{mg}$ of protein was given intravenously immediately after operation. A shielded sodium iodide scintillation detector (crystal diameter $1 \frac{1}{2}$ in $(3.8 \mathrm{~cm})$, thickness 1 in $(2.5$ $\mathrm{cm})$ ) fitted with a cylindrical brass collimator (internal diameter $1 \frac{1}{2}$ in $(3.8 \mathrm{~cm})$, length $\frac{1}{2}$ in. $(1.3 \mathrm{~cm})$ ), using a singlechannel analyser and scaler, was used to count the radiation pulses.

Measurements were made within one hour, and usually within 30 minutes, of injection of the radioisotope. In six patients, however, the first measurement could not be carried out for four to five hours after administration. Counting was performed at $10-\mathrm{cm}$ intervals from the groin crease along both legs. The count rate at each of these points was measured daily, together with the precordial and background count rates, for the next 7-10 days.

\section{Analysis of Data}

The counts from every patient were analysed in three different ways by calculation of the percentage uptake index, the statistical index, and the relative uptake index.

\section{PERCENTAGE UPTAKE INDEX}

The percentage uptake index $(P)$ at each point on the leg was calculated by means of the following equation $P=100$ 
$\left(\mathrm{C}^{1}-\mathrm{B}\right) /\left(\mathrm{H}^{1}-\mathrm{B}\right)$, where $\mathrm{C}^{1}$ is the count rate over any one site on the leg, $\mathrm{H}^{1}$ is the count rate over the precordium, and $B$ is the background count rate. The presence of a thrombus was diagnosed if the difference in percentage uptake between adjacent points on the same leg or corresponding points on the two legs was 15 or more, but only when this difference was maintained for 48 hours.

\section{STATISTICAL INDEX}

In an attempt to avoid the errors inherent in the fixed percentage index owing to the random nature of the counts an alternative method was examined. If a difference in percentage uptake between adjacent or corresponding points exceeded three times the standard deviation of that difference, then this was regarded as significant and indicative of a positive diagnosis. Thus the statistical index (I) was defined as

$$
I=\frac{\text { Difference in percentage uptake between two positions }}{3 \times \text { standard deviation of the difference }}
$$

From this formula it is apparent that any value of $I$ which is greater than 1.0 may be regarded as significant.

\section{RELATIVE UPTAKE INDEX}

In the calculation of the relative uptake index we have attempted to take into account the errors due to the unequal distribution of radioactivity throughout the limbs as well as those due to their random nature. We have made two assumptions in the calculation-(1) that radioactive fibrinogen mixes uniformly with the main mass of circulating fibrinogen so that its distribution is predominantly intravascular throughout the period of the investigation, and (2) that the relative quantity of fibrinogen in any region of the body remains the same throughout the period of the investigation provided no local deposition of fibrinogen occurs.

If the second assumption is correct, then in the absence of a thrombus the ratio of the count rate at any point on the leg to the count rate over the heart may be expected to remain constant. This ratio can be found by carrying out the first measurement soon after the administration of the labelled fibrinogen and before there can have been any significant uptake of radioactivity by a thrombus. In the event of any subsequent uptake of radioactive fibrinogen by a thrombus the ratio at that point will then change and there will be a discrepancy between the observed and expected count rates.

The relative uptake index $(R)$ at any point is defined as the ratio of the difference between the observed and expected count rates to the expected count rate. The expected count rate $(\mathrm{Ce})$ at any point is given by the equation $\mathrm{Ce}=\mathrm{C}_{0} \mathrm{H} / \mathrm{H}_{0}$ $=\mathrm{KH}$, where $\mathrm{C}_{0}$ and $\mathrm{H}_{0}$ are the net count rates measured shortly after the administration of ${ }^{125}$ I-fibrinogen at a point on the leg and at a reference point over the heart respectively, $H$ is the net count rate over the heart at any subsequent time, and $\mathrm{K}$ is a constant equal to $\mathrm{C}_{0} / \mathrm{H}_{0}$. Then by definition $\mathbf{R}=(\mathrm{C}-\mathrm{Ce}) / \mathrm{Ce}$ or $\mathbf{R}=(\mathrm{C}-\mathrm{KH}) / \mathrm{KH}$, where $\mathrm{C}$ is the net count rate observed over any point on the leg at any subsequent time. Let the difference between the observed and expected count rates $(C-K H)$ equal $T$; then $R=T / H$.

If there is no thrombus present $T=0$ and $R=0$. If a thrombus is present, then because $H$ decreases continuously $\mathbf{R}$ will increase continuously if lysis does not occur. Further thrombosis at the same position would augment the rate of increase of $R$, whereas lysis would decrease it. $R$ is thus independent of unequal distribution of the radioisotope along the leg due to anatomical variations and also takes into account the effect of statistical accuracy of the measured count rate. $A$ value of $R$ at any point which is greater than three times the standard deviation, and which increases con- tinuously, may be taken to indicate the presence of a thrombus at that point.

\section{Results}

By means of a relatively simple computer program the values of I for adjacent points on the same leg and corresponding positions on the two legs were calculated daily and were compared with those of $\mathrm{P}$. The computer program also calculated the $R$ values and indicated the points at which a significant increase or decrease had occurred. Of the 41 patients studied, $\mathbf{P}$ gave positive evidence of the presence of thrombosis by the $15 \%$ criterion in 22 .

COMPARISON OF STATISTICAL INDEX AND PERCENTAGE UPTAKE INDEX

Of the 22 patients from whom a $P$ value of $15 \%$ or more was obtained, 9 had a value of I greater than 1.0 on the same day to confirm the diagnosis (Table I). In 13 other patients, however, $I$ indicated the presence of thrombosis at least one day earlier than did $P$. In these 13 patients the average value of $P$ at positions where the value of $I$ was greater than 1.0 was $7.7 \%$, with a range of 3.1 to $14.5 \%$. As shown in Table $I$, in all patients in whom $P$ gave positive results $I$ was also positive. In addition, however, I gave a positive diagnosis in 14 of the 19 patients in whom $P$ had given negative results.

TABLE I-Comparison of Percentage Uptake and Statistical Indices in 41 Patients

\begin{tabular}{|c|c|c|c|}
\hline & & $\begin{array}{c}\text { No. of } \\
\text { Measurement Positions }\end{array}$ & $\begin{array}{l}\text { No. of } \\
\text { Patients }\end{array}$ \\
\hline $\begin{array}{l}\text { Positive by percentage uptake index only } \\
\text { Positive by statistical index only } \ldots \\
\text { Positive by both indices } \quad . .\end{array}$ & $\begin{array}{l}\cdots \\
\cdots\end{array}$ & $\begin{array}{r}3 \\
120 \\
66\end{array}$ & $\begin{array}{r}0 \\
14 \\
22\end{array}$ \\
\hline
\end{tabular}

COMPARISON OF RELATIVE UPTAKE INDEX AND PERCENTAGE UPTAKE INDEX

As pointed out above the value of $\mathbf{R}$ will be zero in the absence of a thrombus but will increase continuously in the presence of a thrombus. The values of $R$ found in a patient in whom no thrombus was diagnosed are shown in Fig 1, and the curve obtained from a patient in whom $\mathbf{P}$ gave a positive diagnosis is shown in Fig 2. Continuously increasing values of $\mathbf{R}$ at two adjacent points on the same patient in whom $P$

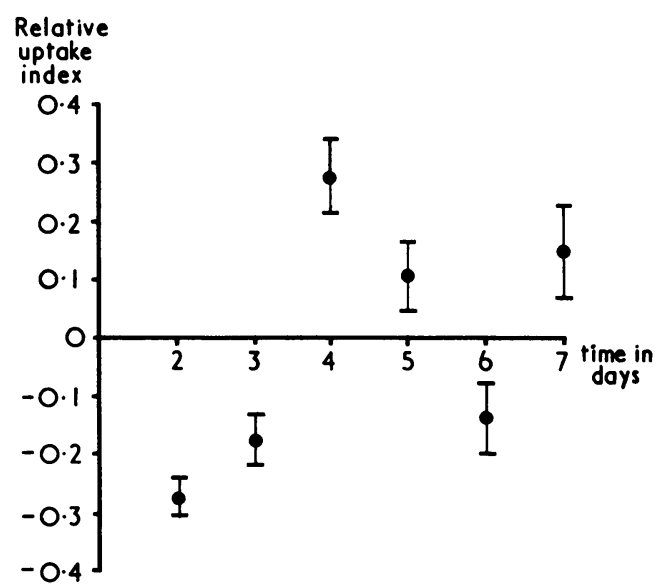

FIG. 1-Daily values ( \pm 1 S.D.) of relative uptake inder at a point where thrombosis did not occur. 


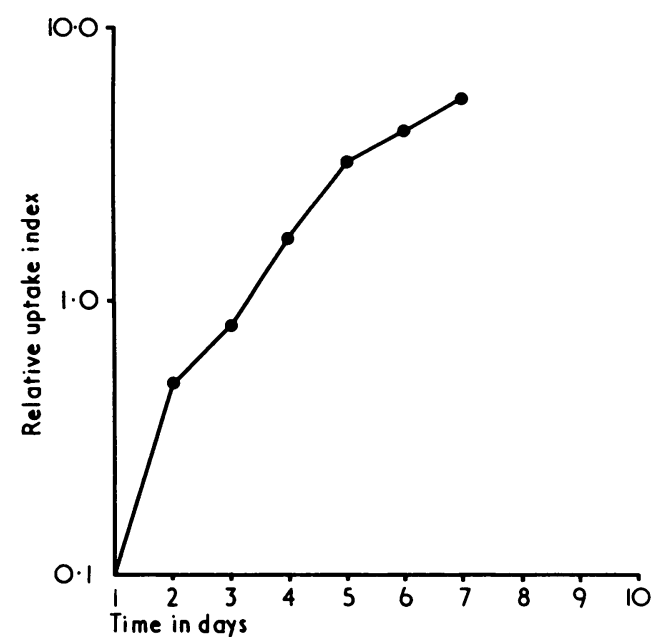

FIG. 2-Variation in value of relative uptake index with time when thrombus is present.

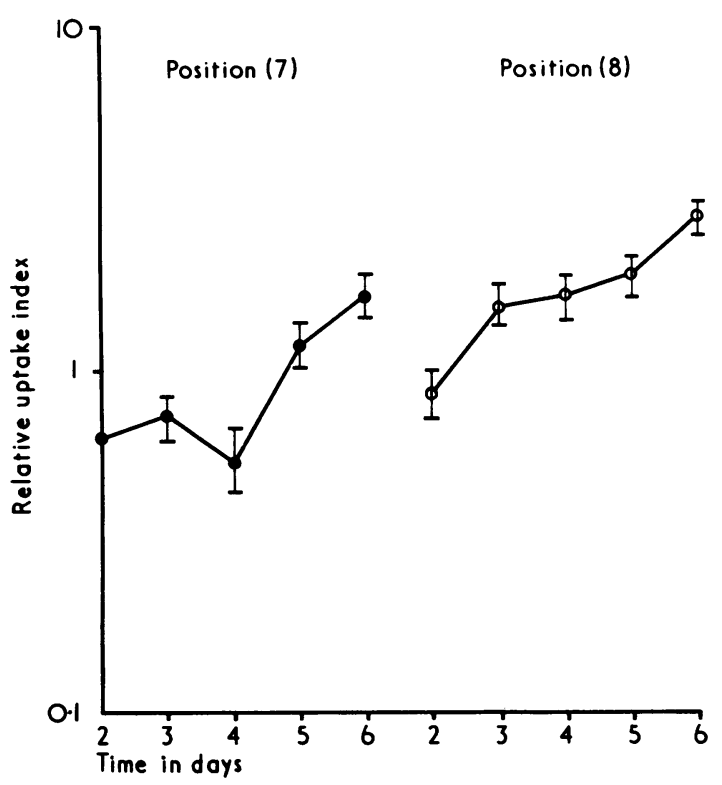

FIG. 3-Daily values ( \pm 1 S.D.) of relative uptake index at two adjacent points (positions 7 and 8 ) on patient in whom percentage uptake index did not show presence of in whom percentage uptake index did not show presence of
thrombus. Relative uptake index suggests a thrombus is present.

did not indicate a positive diagnosis are shown in Fig 3; the shape of the curve at position 8 is explained by the continuing deposition of new thrombus.

The values of $R$ at three adjacent sites are shown in Fig 4. At positions $A$ and $B$ the value of $R$ is increasing, whereas at position $C$ there was an initial rise up to day 4 and the value then fell, indicating that lysis was probably occurring at this point.

In the present studies $P$ gave a positive indication of thrombus in 69 positions (Tables $I$ and II). $R$ gave a positive TABLB II-Comparison of Percentage Uptake and Relative Uptake Indices in
41 Patients

\begin{tabular}{|c|c|c|c|}
\hline & & $\begin{array}{l}\text { No. of } \\
\text { Measurement Positions }\end{array}$ & $\begin{array}{c}\text { No. of } \\
\text { Patients }\end{array}$ \\
\hline $\begin{array}{l}\text { Positive by percentage uptake index only } \\
\text { Positive by relative uptake index only } \\
\text { Positive by both indices } \quad . .\end{array}$ & $\because$ & $\begin{array}{r}17 \\
183 \\
52\end{array}$ & $\begin{array}{r}2 \\
14 \\
20\end{array}$ \\
\hline
\end{tabular}

result at 52 of these positions in 20 patients (Table II). In 8 of these patients bilateral thrombosis was diagnosed by both methods; of the other $12, \mathrm{R}$ showed bilateral thrombosis in only 8 . $R$ gave negative results in two patients in whom $P$ showed evidence of thrombosis at 17 positions. $R$ gave positive results at 183 positions at which $P$ provided no evidence of thrombosis. Of the 20 patients with positive indications by both methods in 11 these were obtained on the same day and in $5 \mathrm{R}$ gave the earlier indication by one to three days. In the other 4 the percentage uptake index gave the earlier indication by one day; but these were among the 6 in whom it had been necessary to delay the first measurement until four to five hours after the administration of the ${ }^{125} \mathrm{I}-$ fibrinogen instead of the usual one hour. Because of this it was not possible to obtain a value of $R$ at the time when $P$ first indicated thrombosis, since the interpretation of $R$ requires the measurement of a rate of increase. However, on the next day in each of these patients $R$ gave a positive indication of thrombosis.

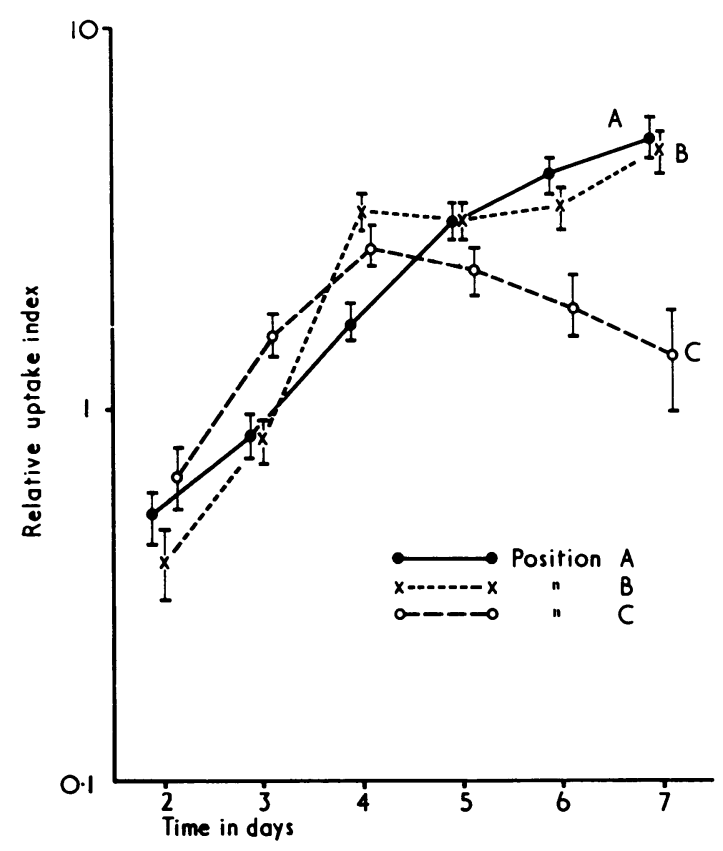

FIG. 4-Daily values ( \pm 1 S.D.) of relative uptake index at three adjacent sites (positions $A, B$, and $C$ ) in patient with venous thrombosis.

\section{Discussion}

The errors in the determination of any radioisotope count depend on the total number of measured counts or, when a rate meter is used, on both the count rate and time constant used. In practice it is seldom practicable to accumulate counts over one position for more than 30 seconds owing to the number of patients who require to be investigated in one day. It is therefore essential in the interpretation of the data that account be taken of these counting errors.

The percentage uptake index, whether it relies on a difference of $15 \%$ or $20 \%$ (Negus et al., 1968; Pai and Negus, 1971), is open to criticism because it does not take account of the count rate errors. In addition the criteria were established by comparison with a phlebographic technique, which is probably a less sensitive indicator of the presence of thrombus than the radioisotope method (Browse et al., 1971). The statistical index, which does take account of counting errors, was positive in all cases diagnosed by the percentage uptake criterion in the present study and also gave a positive indication in several other patients. It seems likely that if a 
percentage uptake criterion is to be used a more realistic figure for a significant difference would be $8 \%$ rather than $15 \%$ with the usual collimator employed. However, despite the larger number of positive indications obtained with the statistical index than with the percentage uptake index, both these indices fail to take account of the unequal distribution of fibrinogen in the blood and tissue of the limbs at adjacent points.

The use of the relative uptake index, on the other hand, avoids the necessity of having to assess the significance of counts at any one point on a leg by reference to those made at another. It is interesting that it gave not only a number of positive results in addition to those given by the percentage uptake index but also a number of negative results where the latter was positive. This was not entirely unexpected, since anatomical variations in legs are such that when only one value $(15 \%)$ is used for significance the percentage uptake index is most unlikely to give correct results for all points on all patients.

Phlebographic confirmation of our results was not carried out since, as noted above, the standardization of a technique by one which is less sensitive is regarded as unhelpful.

The use of the relative uptake index avoids the application of the same arbitrary limits for a positive diagnosis to all patients. The reported incidence of venous thrombosis detected by ${ }^{1: 2}$ I-fibrinogen is of the order of $30 \%$ (Flan= et al., 1968; Negus et al., 1968; Hills el al., 1972). On the basis of the results reported here this seems to us to be a low estimate. We have shown that the sensitivity of the method is increased when account is taken of variations in count rates and anatomy, so that the relative uptake index may well provide a more accurate indicator of the presence of deep vein thrombosis than those in current use. Caution must be advised, however, in estimating the incidence of thrombosis from the data reported here, because the patients studied were selected specially to give a high number of positive results.

The method of counting does not differ from that commonly used, and the mathematical analysis is simple and $\stackrel{\vec{\rho}}{\rightarrow}$ suitable for routine use. By using the program daily results can $\overline{0}$ be obtained a few minutes after the data have been fed $\underline{\bar{O}}$ into the computer. The computer program will also draw $\frac{\bar{s}}{\frac{1}{T}}$ attention to anatomical points at which a significant change $\stackrel{\mathbb{\Omega}}{\Omega}$ has occurred; this form of data processing saves a great deal of time both in the calculation and in the interpretation of the results.

\section{References}

Atkins, P., and Hawkins, L. A. (1965). Lancet, 2, 1217.

Atkins, P., and Hawkins, L. A. (1968). British fournal of Surgery, 55, 825. Browse, N. L., et al. (1971). British Medical fournal, 4, 325.

Flanc, C., Kakkar, V. V., and Clarke, M. B. (1968). British fournal of î

Surgery, 55, 742.
Hills, N. H., Pflug, J. J., Jeyasingh, K., Boardman, L., and Calnan, J. S. (1972). British Medical fournal, 1, 131 .

Negus, D., Pinto, D. J., Le Quesne, L. P., Brown, N., and Chapman, M. N (1968). British Fournal of Surgery, 55, 835.

Pai, B. Y., and Negus, D. (1971). Lancet, 2, 1098. of Surgery, 7, 215.

\title{
Assessment of Upper Airways Obstruction
}

\author{
D. W. EMPEY
}

British Medical fournal, 1972, 3, 503-505

\begin{abstract}
Summary
An indication of obstruction to the upper airways (trachea and larynx) may be obtained by calculating the ratio of the forced expired volume in one second to the peak expiratory flow rate $\left(F E V_{1} /\right.$ PEFR). This index was found to be usually less than 10 in normal subjects (mean 7.3), and in patients with asthma (mean 6.9), chronic bronchitis (mean 7.7), or interstitial lung disease (mean 6.3). A study of simulated upper airways obstruction showed that this index rises as the obstruction becones more severe. All of 18 patients with proved upper airways obstruction had $\mathrm{FEV}_{1} /$ PEFR indices greater than 10 (mean 14.0). This test can be carried out with forced expiratory manoeuvres only, and it does not require the use of complicated equipment. An FEV $/$ PEFR ratio greater than 10, when upper airways obstruction is suspected, indicates that significant obstruction may be present. High values suggest that the obstruction may be severe, and that further investigations are indicated.
\end{abstract}

Department of Chest Medicine, London Hospital, London E.1 D. W. EMPEY, M.B., M.R.C.P., Wellcome Research Fellow

\section{Introduction}

Lesions of the upper airways, such as tracheal stenosis or laryngeal tumours, are relatively uncommon compared with obstruction of the lower airways, such as occurs in asthma and chronic bronchitis. It is, however, important to recognize upper airways obstruction, especially as the patients may go into respiratory failure (Bland et al., 1969; Clark, 1970; Hughes et al., 1972). Frequently neither the nature nor the severity of the $\tilde{\sigma}$ lesion is realized clinically unless endoscopy is performed. I Conventional lung function tests may not fully disclose the extent of the obstruction, and for this reason more complex $N$ tests have been devised to differentiate between upper and lower airways obstruction. The most satisfactory of these involves the construction of flow-volume curves (Jordanoglou and Pride, 1968). Expensive equipment is required for this, however, and the results depend to some extent on the cooperation and effort of the patient.

Attempts have previously been made to obtain by indirect means some of the information given by flow-volume curves, but these methods suffer from disadvantages similar to those involved in constructing actual flow-volume curves (Nairn and McNeill, 1963; Clark, 1970). Theoretical considerations (see below) suggest that it might be possible to identify patients with upper airways obstruction on the basis of forced expiratory flow tests using readily available equipment-namely, a dry spirometer and a Wright peak flow meter. The present study was designed to test this hypothesis by comparing the results obtained from subjects with real and simulated upper airways obstruction with those from normal subjects and patients with lower airways obstruction or interstitial lung disease. 